\title{
Anomalous dynamics of aqueous solutions of di-propylene glycol methylether confined in MCM-41 by quasielastic neutron scattering
}

\author{
Jan Swenson, ${ }^{1, \text { a) }}$ Khalid Elamin, ${ }^{1}$ Guo Chen, ${ }^{1}$ Wiebke Lohstroh, ${ }^{2}$ \\ and Victoria Garcia Sakai ${ }^{3}$ \\ ${ }^{1}$ Department of Applied Physics, Chalmers University of Technology, SE-412 96 Göteborg, Sweden \\ ${ }^{2}$ Heinz Maier-Leibnitz Zentrum (MLZ), Technische Universität München, Lichtenbergstr. \\ 1, 85748 Garching, Germany \\ ${ }^{3}$ ISIS Facility, Rutherford Appleton Laboratory, Chilton, Didcot, OX11 OQX Oxfordshire, United Kingdom
}

(Received 13 August 2014; accepted 10 November 2014; published online 1 December 2014)

\begin{abstract}
The molecular dynamics of solutions of di-propylene glycol methylether (2PGME) and $\mathrm{H}_{2} \mathrm{O}$ (or $\mathrm{D}_{2} \mathrm{O}$ ) confined in $28 \AA$ pores of MCM-41 have been studied by quasielastic neutron scattering and differential scanning calorimetry over the concentration range $0-90 \mathrm{wt} . \%$ water. This system is of particular interest due to its pronounced non-monotonic concentration dependent dynamics of 2PGME in the corresponding bulk system, showing the important role of hydrogen bonding for the dynamics. In this study we have elucidated how this non-monotonic concentration dependence is affected by the confined geometry. The results show that this behaviour is maintained in the confinement, but the slowest diffusive dynamics of 2PGME is now observed at a considerably higher water concentration; at $75 \mathrm{wt} . \%$ water in MCM-41 compared to $30 \mathrm{wt} . \%$ water in the corresponding bulk system. This difference can be explained by an improper mixing of the two confined liquids. The results suggest that water up to a concentration of about $20 \mathrm{wt} . \%$ is used to hydrate the hydrophilic hydroxyl surface groups of the silica pores, and that it is only at higher water contents the water becomes partly mixed with 2PGME. Hence, due to this partial micro-phase separation of the two liquids larger, and thereby slower relaxing, structural entities of hydrogen bonded water and 2PGME molecules can only be formed at higher water contents than in the bulk system. However, the $Q$-dependence is unchanged with confinement, showing that the nature of the molecular motions is preserved. Thus, there is no indication of localization of the dynamics at length scales of less than $20 \AA$. The dynamics of both water and 2PGME is strongly dominated by translational diffusion at a temperature of 280 K. (C) 2014 AIP Publishing LLC. [http://dx.doi.org/10.1063/1.4902250]
\end{abstract}

\section{INTRODUCTION}

It is well known that the special properties of hydrogen bonds give hydrogen bonded liquids in general, and water in particular, their anomalous structural and dynamical properties. In fact, it is the intermediate strength of the hydrogen bond that makes life, as we know it, possible. Therefore, it is not an exaggeration to state that it is of extraordinary importance to understand the role of hydrogen bonds in the structural and dynamical properties of hydrogen bonded liquids.

Different approaches have been used to perform systematic studies of hydrogen bonds and their influence on liquid properties. For instance, binary liquids, where one or both components display hydrogen bonding, have been used to systematically vary the density of hydrogen bonds in the system. Such studies have shown that the concentration dependence of the molecular dynamics is strongly dependent on how the hydrogen bonding changes over the concentration range. As an example we can compare the concentration dependent glass transitions, $\mathrm{T}_{\mathrm{g}}$, of aqueous solutions of propylene glycol (PG) and propylene glycol methylether (PGME), respectively. $\mathrm{PG}$ has two $\mathrm{OH}$ end groups enabling the formation of chains of hydrogen bonded molecules, whereas

\footnotetext{
a) Author to whom correspondence should be addressed. Electronic mail: jan.swenson@chalmers.se
}

PGME that has only one $\mathrm{OH}$ end group can only form pairs of two molecules. In fact, this has been clearly indicated by comparing structural relaxation times of the oligomeric $n$ PGME-series to those of $n$-propylene glycol dimethyl ethers ( $n$ PGDE), which cannot form hydrogen bonds. ${ }^{1,2}$ The difference in density of hydrogen bonds between PG and PGME also gives rise to a difference in $\mathrm{T}_{\mathrm{g}}$ (168 $\mathrm{K}$ for $\mathrm{PG}$ compared to $143 \mathrm{~K}$ for PGME). Upon increasing water content the $\mathrm{T}_{\mathrm{g}}$ of PG decreases slowly, but steadily, until partial crystallisation of water occurs at about 60 wt. $\%$ water. ${ }^{3}$ On the other hand, for PGME the concentration dependence of $\mathrm{T}_{\mathrm{g}}$ exhibits an anomalous behavior, with a maximum of $169 \mathrm{~K}$ at $55 \mathrm{wt} . \%$ water. ${ }^{3}$ Clearly, the hydrogen bonds in water are affecting the viscosity and glass transition related dynamics in these systems very differently. In the case of the PG-water system infinitely long chains or clusters of hydrogen bonded molecules can be formed at all water concentrations, and therefore it is likely that water does not substantially affect the dynamics of the PG molecules. Only a slight plasticizing effect of added water can be observed, similar to what is typically observed for aqueous solutions. For PGME, previous studies have indicated that water molecules form hydrogen bonded bridges between two or more PGME molecules, leading to the formation of larger structural entities than single pairs of PGME molecules. ${ }^{3,4}$ The increase in size of these water mediated 
clusters of hydrogen bonded molecules should then be responsible for the increase of $T_{g}$. However, there is obviously a limit to how large these clusters can grow. At high water concentrations the water molecules must surround the PGME molecules and give rise to a plasticizing effect. ${ }^{4}$

Similar non-monotonic concentration dependences of viscosity related dynamics have been observed for alcoholwater mixtures, ${ }^{5-7}$ although in these systems they have been attributed to the hydrophobic interaction between alcohol molecules and surrounding water molecules. ${ }^{8-11}$ This gives rise to both slower water dynamics ${ }^{12-14}$ and a higher local average density ${ }^{8-11}$ of the two molecular water layers closest to the alcohol molecules. In fact, the local density of the water and its molecular dynamics seems to be strongly related. ${ }^{15}$ The findings for alcohol-water mixtures further imply that it is mainly the dynamics of water that exhibits an anomalous nonmonotonic concentration dependence, in contrast to what was observed for the 2PGME-water system. ${ }^{16}$

Further insights about how hydrogen bonds affect the structure and dynamics of liquids, and the possible formation of larger structural entities of hydrogen bonded molecules, can be gained by imposing geometrical constraints. The formation of hydrogen bonded clusters that are larger than the available space are thus not possible. The interaction between the surface of the confining material and the confined liquid will also influence the ability to form hydrogen bonds in the vicinity to the surface. Thus, a deeper understanding of hydrogen bonds, and how they affect the structural and dynamical properties of liquids, can be obtained from confinement studies. Moreover, confined liquids in general and confined water in particular are of central importance in a wide range of applied fields, in, e.g., biology, geology, and many technologies, ${ }^{17}$ which further justifies such studies. In what follows we investigate how the dynamics of 2PGME-water solutions is altered in $28 \AA$ pores of MCM- 41 . We have used quasielastic neutron scattering (QENS) and differential scanning calorimetry (DSC) to explore how the molecular dynamics and $\mathrm{T}_{\mathrm{g}}$ of the corresponding bulk solutions are altered by the confined geometry. The results show that a non-monotonic concentration dependence is maintained in this confinement, but the concentration where the slowest dynamics of 2PGME is obtained is shifted to a substantially higher water concentration. This shift can be explained by an assumption that water prefers to form hydrogen bonds to the hydrophilic silica surface, causing a partial micro-phase separation of the two liquids. It is not until the silica surface is fully hydrated that additional water can bridge between two 2PGME molecules. A similar micro-phase separation of binary liquids has previously been experimentally observed for other solutions confined in MCM-41, ${ }^{18,19}$ although molecular dynamics simulations of aqueous solutions have shown the opposite behavior with the alcohol or sugar molecules hydrogen bonded to the silica surface..$^{20,21}$

\section{EXPERIMENTAL}

\section{A. Sample preparations}

For this study, fully protonated 2PGME, purchased from Sigma-Aldrich and freeze dried to remove small residual impurities, ${ }^{22}$ was mixed with distilled milli-Q $\mathrm{H}_{2} \mathrm{O}$ or $\mathrm{D}_{2} \mathrm{O}$ (from Larodan Fine Chemicals) to produce bulk solutions containing $0,15,30,45,60,75$, and 90 wt. $\%$ water in the case of $\mathrm{H}_{2} \mathrm{O}$. The solutions with $\mathrm{D}_{2} \mathrm{O}$ were prepared such that the molar ratio of water to $2 \mathrm{PGME}$ was the same as for the solutions with $\mathrm{H}_{2} \mathrm{O}$. To ensure homogenous mixing of the two liquids each solution was put in an ultrasonic bath for $30 \mathrm{~min}$.

The silica matrix MCM-41, with an average pore diameter of $28 \AA$, was purchased from Sigma-Aldrich and dried in a vacuum oven at $150{ }^{\circ} \mathrm{C}$ for two days to remove internal and external surface water. The 2PGME-water solutions were then confined in the dried MCM-41 by soaking the powder directly into the corresponding bulk solutions under vacuum. After the pores had been filled with the bulk solutions the powder was removed from the bulk solutions and gently dried by a tissue before the samples were measured by QENS.

\section{B. DSC measurements}

DSC measurements were performed on the TA Instruments DSC Q1000. The solution filled MCM-41 samples were placed in hermetically sealed aluminium pans. The glass transition temperature $\left(T_{g}\right)$ of each sample was determined at a heating rate of $10 \mathrm{~K} \mathrm{~min}^{-1}$ after the sample had been cooled rapidly from $313 \mathrm{~K}$ to $123 \mathrm{~K}$ at a rate of $-30 \mathrm{~K} \mathrm{~min}^{-1}$. The $T_{g}$ value was defined as the low temperature onset of the step in heat capacity. Three samples of each concentration were measured to ensure repeatability and to determine the sampleto-sample variability, which was used to estimate the experimental errors of the determined $T_{g}$-values.

\section{QENS measurements}

The QENS measurements were carried out at $280 \mathrm{~K}$ on the cold neutron time-of-flight spectrometer TOFTOF at the neutron scattering facility FRMII, Heinz Maier-Leibnitz Zentrum in Garching, Germany. However, due to a problem with the measurement of the sample containing $75 \mathrm{wt} . \% \mathrm{H}_{2} \mathrm{O}$ in the solution, this sample and the corresponding sample containing $\mathrm{D}_{2} \mathrm{O}$ (for comparison with the $\mathrm{H}_{2} \mathrm{O}$ sample and the TOFTOF data on the same sample) were remeasured on the IRIS spectrometer at the pulsed neutron spallation source ISIS at the Rutherford Appleton Laboratory, United Kingdom. The same energy resolution was used and the corresponding bulk solutions were earlier measured on this instrument. ${ }^{16}$ The TOFTOF spectrometer is described in detail in Ref. 23 and the IRIS spectrometer in Ref. 24, so here we will only give some specific details of relevance to the TOFTOF measurements. Using an incident neutron wavelength of $10 \AA$, an energy resolution of $17.5 \mu \mathrm{eV}$ [full width at half maximum (FWHM)] and a total energy window of $\pm 0.7 \mathrm{meV}$ were obtained. The samples were placed in flat aluminium containers with an internal thickness of about $0.3 \mathrm{~mm}$, to obtain a sample transmission of less than 0.9 , so that multiple scattering can be ignored to a good approximation. The raw time-of-flight data were normalized to the incoming flux, the empty can signal was subtracted, and then normalized with respect to a vanadium standard scatterer. The energy transfer and momentum 
transfer $Q$ were calculated. The detectors, each corresponding to a specific scattering angle and therefore also a specific $Q$ value at zero energy transfer, were grouped into 9 groups with $Q$-values from $0.2 \AA^{-1}$ to $1.0 \AA^{-1}$, in steps of $0.1 \AA^{-1}$. Due to the finite energy resolution of the spectrometer the corrected measured spectra $I_{m}(Q, \omega)$ are actually convolutions of the scattering law $S(Q, \omega)$ with the resolution function $R(Q, \omega)$ of the instrument, as determined by the scattering from the vanadium plate in the same experimental geometry, i.e., $I_{m}(Q, \omega)$ $=S(Q, \omega) \otimes R(Q, \omega)$. However, if $I_{m}(Q, \omega)$ is Fourier transformed from energy to time, the convolution is reduced to a simple multiplication in the time domain, i.e., $I_{m}(Q, t)=I(Q, t)$ $\times R(Q, t)$. The intermediate scattering function $I(Q, t)$ is then easily obtained by dividing the Fourier transformed data with the Fourier transform of the resolution function, i.e., $I(Q, t)$ $=I_{m}(Q, t) / R(Q, t)$.

The reason for why two isotopically different samples (containing either $\mathrm{H}_{2} \mathrm{O}$ or $\mathrm{D}_{2} \mathrm{O}$ ) of each water concentration were measured is the possibility to use the different scattering cross-sections of $\mathrm{H}$ and $\mathrm{D}$ to distinguish between the water dynamics and the motion of 2PGME molecules. The total scattering cross-section of $\mathrm{H}$ is at least one order of magnitude larger than any other chemical component, including D. This implies that it is mainly the motion of H-atoms that is probed in a material with a reasonably high hydrogen content. Moreover, the scattering from $\mathrm{H}$ is mainly incoherent, which means that it is basically the self-correlations in space and time that are probed in the QENS measurements. Thus, except for the water rich samples containing 75 and 90 wt.\% water, a replacement of $\mathrm{H}_{2} \mathrm{O}$ by $\mathrm{D}_{2} \mathrm{O}$ causes the water dynamics to be almost invisible and mainly the self-motions of the $\mathrm{H}$-atoms in 2PGME are probed. However, for the two highest water contents the number of $\mathrm{H}$ atoms in 2PGME is not sufficiently large to completely ignore the scattering from $\mathrm{D}_{2} \mathrm{O}$, particularly not for the sample with $90 \mathrm{wt} . \% \mathrm{D}_{2} \mathrm{O}$, where water contributes with almost $30 \%$ to the total scattering of the confined solution in the measured $Q$-range $<1 \AA^{-1}$.

The scattering contribution from the water was extracted by subtracting $I(Q, t)$ of confined $2 \mathrm{PGME}$ in $\mathrm{D}_{2} \mathrm{O}$ from that of confined 2PGME in $\mathrm{H}_{2} \mathrm{O}$, after each intermediate scattering function had been weighted by the total scattering cross section of the sample at that particular Q-value. Thereafter, the resulting $I(Q, t)$ was renormalized to unity at $\mathrm{t}=0$. Here it should be noted that in the $2 \mathrm{PGME}-\mathrm{D}_{2} \mathrm{O}$ mixtures the $\mathrm{H}$ atom of the single $\mathrm{OH}$ group of each 2PGME molecule is rapidly exchangeable with $\mathrm{D}$ atoms in $\mathrm{D}_{2} \mathrm{O}$. As this is only one in 16 of each 2PGME molecule we expect the influence to be minor on the estimated water dynamics. To handle the nonnegligible scattering from $\mathrm{D}_{2} \mathrm{O}$, particularly at the two highest water concentrations, its scattering contribution was included when the scattering contribution from 2PGME was subtracted from the total scattering of the corresponding $2 \mathrm{PGME}-\mathrm{H}_{2} \mathrm{O}$ sample. Hence, not only the scattering from 2PGME was subtracted but also a small fraction of the scattering from water, i.e., only the difference in total scattering from $\mathrm{H}_{2} \mathrm{O}$ and $\mathrm{D}_{2} \mathrm{O}$ remained (we made no distinguishing between coherent and incoherent scattering in this case), which anyhow should be enough to determine the water dynamics to a good approximation.

\section{RESULTS}

Figure 1 shows DSC data of the for 2PGME-water solutions confined in $28 \AA$ pores of MCM-41. In the inset the concentration dependence of $T_{g}$ is compared with the corresponding $T_{g}$ of the bulk solutions. ${ }^{16}$ It can be seen that $T_{g}$ of confined 2PGME is slightly higher than for bulk 2PGME, but in the concentration range up to about 45 wt. $\%$ water the $T_{g^{-}}$ value of the confined solutions remains basically the same, within the experimental errors, whereas it increases by more than $15 \mathrm{~K}$ for the bulk solutions. The increase of $T_{g}$ for the bulk solutions can be explained by the formation of larger structural (and relaxing) entities, when water form hydrogen bonded bridges between two 2PGME molecules, as discussed above. ${ }^{4}$ The nearly constant $T_{g}$ of the confined solutions in this concentration range then indicates that no growth of such structural entities occur. In fact, since no plasticizing effect of water is observed it seems questionable whether the two liquids are even mixed in this concentration range of the confined solutions. Although the corresponding bulk solutions are homogenously mixed, previous studies of solutions confined in the silica pores of MCM-41 have indicated ${ }^{18,19}$ that the hydrophilic hydroxyl surface groups of the silica pores may induce a micro-phase separation of the two liquids due to a preference of one of the liquids to coordinate to these surface groups. This would then make $T_{g}$ (which is caused by the freezing-in of the structural relaxation of 2PGME on a time scale of $100 \mathrm{~s}$ ) basically concentration independent until the water content is sufficiently high to partly mix water with 2PGME. This model of partial micro-phase separation is also consistent with the shift of the $T_{g}$-maximum from the bulk solution with $45 \mathrm{wt}$. $\%$ water to the confined solution with 75 wt. $\%$ water. Hence, this shift suggests that the large water bridged entities are formed at higher water concentrations.

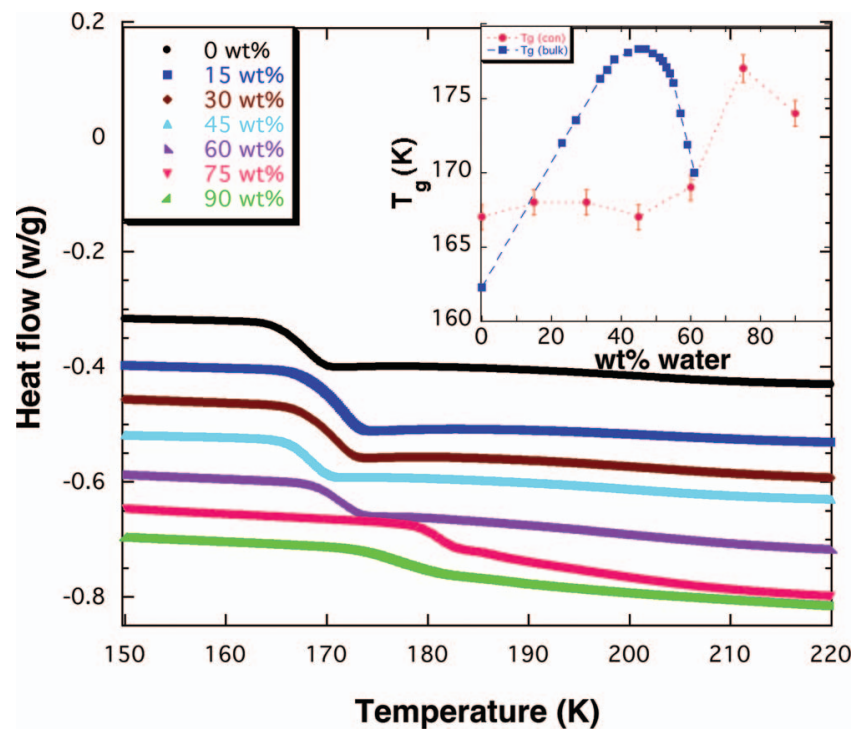

FIG. 1. DSC data of the 2PGME-water solutions confined in $28 \AA$ pores of MCM-41. The inset shows the concentration dependence of the glass transition temperature, $T_{g}$, of the confined 2PGME-water solutions (filled circles), in comparison with $T_{g}$ of the non-freezing bulk solutions (filled squares), from Ref. 16. 


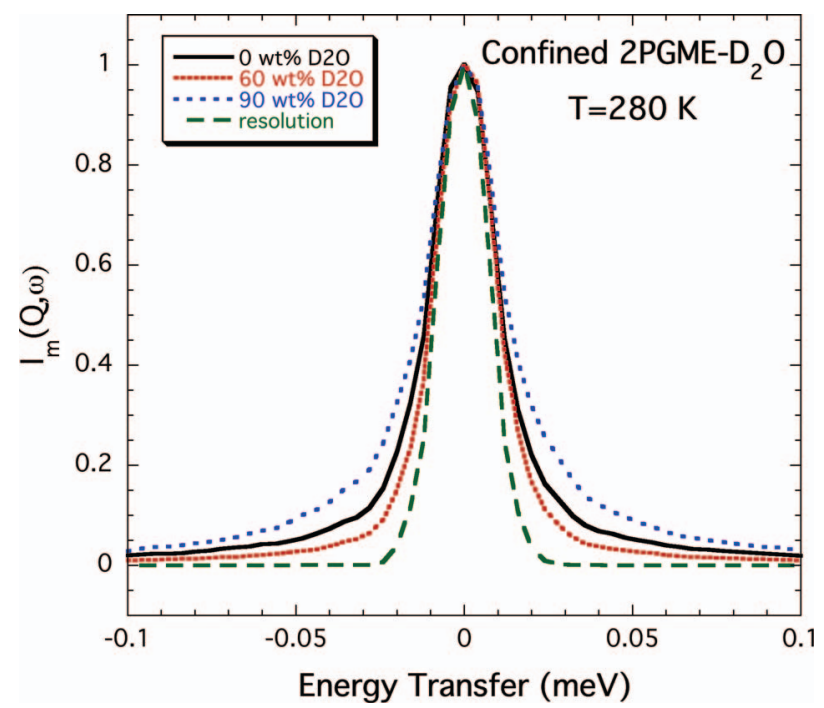

FIG. 2. Quasielastic neutron scattering spectra, $I_{m}(Q, \omega)$, at $280 \mathrm{~K}$ and $Q=0.70 \AA^{-1}$ for confined 2PGME- $\mathrm{D}_{2} \mathrm{O}$ solutions containing 0,60 , and 90 wt.\% water, as indicated in the figure. In addition, a resolution measurement of vanadium is included to show the instrumental energy resolution (FWHM $=17.5 \mu \mathrm{eV}$ ) of the spectrometer. For ease of comparison, all spectra are normalized to unity at zero energy transfer.

In Fig. 2 measured spectra of confined 2PGME- $\mathrm{D}_{2} \mathrm{O}$ with 0,60 , and $90 \mathrm{wt} . \%$ water are shown for $\mathrm{T}=280 \mathrm{~K}$ and $Q=0.70 \AA^{-1}$, together with the vanadium resolution measurement for comparison (the peak maximum of each spectrum was normalized to unity). Since it is samples containing $\mathrm{D}_{2} \mathrm{O}$, the main contribution to the scattering comes from 2PGME. From the figure it is evident that the quasielastic broadening exhibits a non-monotonic concentration dependence. The broadening decreases, i.e., the dynamics becomes slower, when the water content increases from $0 \mathrm{wt} . \%$ to 60 wt. \%, but at the highest water contents the broadening increases rapidly with increasing water concentration, and the fastest dynamics is observed for the sample containing 90 wt. $\%$ water.

This non-monotonic concentration dependence of the dynamics is also evident after the measured spectra have been Fourier transformed to the intermediate scattering function $I(Q, t)$, which is the data representation we have chosen for the quantitative analysis. Fig. 3 shows $I(Q, t)$ at $Q=0.70 \AA^{-1}$ for confined 2PGME- $\mathrm{H}_{2} \mathrm{O}$ and 2PGME- $\mathrm{D}_{2} \mathrm{O}$ of different water fractions. In addition to the non-monotonic concentration dependences of samples containing both $\mathrm{H}_{2} \mathrm{O}$ and $\mathrm{D}_{2} \mathrm{O}$ it is evident that each relaxation function is stretched in time and therefore best described by a single Kohlrausch-WilliamsWatts (KWW) stretched exponential function, ${ }^{25}$

$$
I(Q, t)=0.85 \cdot \exp \left[-\left(\frac{t}{\tau}\right)^{\beta}\right]+0.15 \quad(0<\beta \leq 1),
$$

where $\tau$ and $\beta$ are the characteristic relaxation time and the stretching parameter, respectively. $I(Q, t)$ is normalized to unity at $\mathrm{t}=0$, but since the scattering contribution from the silica matrix, which was not subtracted, is not negligible $I(Q, t)$ is not expected to decay to zero at long times. Instead, a "background level" corresponding to this elastic scattering

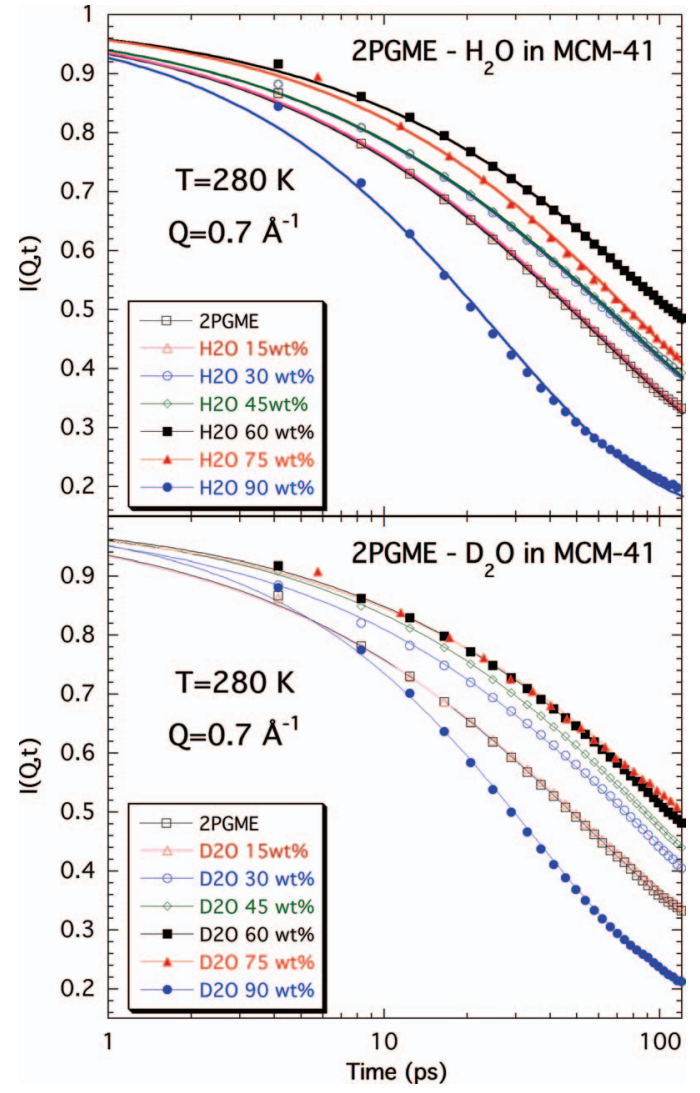

FIG. 3. Intermediate scattering functions, $I(Q, t)$, at $280 \mathrm{~K}$ and $Q=0.70 \AA^{-1}$ for 2PGME-water solutions confined in $28 \AA$ pores of MCM-41. Water weight fractions are shown in the figures. The upper and lower panels show confined 2PGME-water samples containing $\mathrm{H}_{2} \mathrm{O}$ and $\mathrm{D}_{2} \mathrm{O}$, respectively. The solid lines are KWW fits (Eq. (1)) to the experimental data points.

contribution is reached. In the analysis we have made the approximation that this level is independent of both sample and $Q$-value. This is not fully correct, but a reasonable approximation in our case when this "background level" can be experimentally determined, to about 0.15 as shown in Eq. (1), only for a few samples at the highest $Q$-value of $1 \AA^{-1}$. Furthermore, no substantial differences in the relaxation times are obtained if this "background level" is changed to 0.1 or 0.2 , although the fits to the experimental data become worse in the cases when it is clear that the chosen "background level" is inappropriate. It should also be noted that in reality several different types of relaxation processes are expected to occur, such as fast rotations of single water molecules and slower translational diffusion of 2PGME molecules, but from the experimental data it is clear that their time scales are not sufficiently different to distinguish them from each other. Instead, only an average relaxation time, $\langle\tau\rangle$, as a function of $Q$ is obtained from the KWW parameters $\tau$ and $\beta$ by the relation, ${ }^{26}$

$$
\langle\tau\rangle=\frac{\tau}{\beta} \Gamma\left(\frac{1}{\beta}\right),
$$

where $\Gamma$ is the gamma function.

The stretching parameter $\beta$ is both sample and $Q$ dependent. Generally, $\beta$ is in the range $0.4-0.5$ at the lowest $Q$-value of $0.2 \AA^{-1}$ and increases up to $0.65-0.9$ at the highest 
TABLE I. Diffusion constants D and values of $\tau$, $\langle\tau\rangle$, and $\beta$ at $Q=0.70 \AA^{-1}$ are given for the different samples at $T=280 \mathrm{~K}$. Estimated values for the internal water are also provided.

\begin{tabular}{|c|c|c|c|c|}
\hline Sample & $\mathrm{D}\left(10^{-10} \mathrm{~m}^{2} / \mathrm{s}\right)$ & $\tau(\mathrm{ps})$ & $\langle\tau\rangle(\mathrm{ps})$ & $\beta$ \\
\hline 2PGME in MCM-41 & $2.5 \pm 0.3$ & $58 \pm 3$ & $83 \pm 6$ & $0.62 \pm 0.03$ \\
\hline 2PGME with 15 wt. $\% \mathrm{D}_{2} \mathrm{O}$ in MCM-41 & $2.4 \pm 0.3$ & $59 \pm 4$ & $87 \pm 8$ & $0.61 \pm 0.03$ \\
\hline 2PGME with 30 wt. $\% \mathrm{D}_{2} \mathrm{O}$ in MCM-41 & $1.6 \pm 0.2$ & $90 \pm 4$ & $130 \pm 7$ & $0.62 \pm 0.03$ \\
\hline 2PGME with 45 wt. $\% \mathrm{D}_{2} \mathrm{O}$ in MCM-41 & $1.4 \pm 0.2$ & $106 \pm 4$ & $146 \pm 7$ & $0.65 \pm 0.02$ \\
\hline 2PGME with 60 wt. $\% \mathrm{D}_{2} \mathrm{O}$ in MCM-41 & $1.1 \pm 0.2$ & $130 \pm 5$ & $183 \pm 9$ & $0.64 \pm 0.02$ \\
\hline 2PGME with 75 wt. $\% \mathrm{D}_{2} \mathrm{O}$ in MCM-41 & $0.95 \pm 0.2$ & $164 \pm 5$ & $224 \pm 10$ & $0.65 \pm 0.02$ \\
\hline 2PGME with 90 wt. $\% \mathrm{D}_{2} \mathrm{O}$ in MCM-41 & $5.2 \pm 0.5$ & $34 \pm 1$ & $39 \pm 2$ & $0.80 \pm 0.02$ \\
\hline 2PGME with 15 wt. $\% \mathrm{H}_{2} \mathrm{O}$ in MCM-41 & $2.4 \pm 0.3$ & $59 \pm 3$ & $85 \pm 6$ & $0.63 \pm 0.03$ \\
\hline 2PGME with 30 wt. $\% \mathrm{H}_{2} \mathrm{O}$ in MCM-41 & $1.9 \pm 0.3$ & $72 \pm 3$ & $109 \pm 6$ & $0.60 \pm 0.03$ \\
\hline 2PGME with 45 wt. $\% \mathrm{H}_{2} \mathrm{O}$ in MCM-41 & $1.8 \pm 0.3$ & $75 \pm 3$ & $114 \pm 6$ & $0.60 \pm 0.03$ \\
\hline 2PGME with 60 wt. $\% \mathrm{H}_{2} \mathrm{O}$ in MCM-41 & $1.2 \pm 0.4$ & $120 \pm 5$ & $173 \pm 10$ & $0.62 \pm 0.03$ \\
\hline 2PGME with 75 wt. $\% \mathrm{H}_{2} \mathrm{O}$ in MCM-41 & $1.6 \pm 0.3$ & $92 \pm 3$ & $124 \pm 5$ & $0.66 \pm 0.02$ \\
\hline 2PGME with 90 wt. $\% \mathrm{H}_{2} \mathrm{O}$ in MCM-41 & $5.3 \pm 0.6$ & $35 \pm 1$ & $39 \pm 2$ & $0.82 \pm 0.02$ \\
\hline Water in sample with $15 \mathrm{wt} . \%$ water & $3.2 \pm 0.5$ & $50 \pm 5$ & $61 \pm 10$ & $0.73 \pm 0.05$ \\
\hline Water in sample with $30 \mathrm{wt} . \%$ water & $3.1 \pm 0.5$ & $45 \pm 5$ & $65 \pm 12$ & $0.62 \pm 0.05$ \\
\hline Water in sample with $45 \mathrm{wt} . \%$ water & $3.2 \pm 0.5$ & $42 \pm 4$ & $62 \pm 10$ & $0.61 \pm 0.05$ \\
\hline Water in sample with $60 \mathrm{wt} . \%$ water & $1.4 \pm 0.5$ & $109 \pm 8$ & $155 \pm 22$ & $0.63 \pm 0.05$ \\
\hline Water in sample with $75 \mathrm{wt} . \%$ water & $3.3 \pm 0.6$ & $46 \pm 4$ & $60 \pm 8$ & $0.69 \pm 0.04$ \\
\hline Water in sample with $90 \mathrm{wt} . \%$ water & $5.3 \pm 0.8$ & $35 \pm 3$ & $38 \pm 5$ & $0.86 \pm 0.03$ \\
\hline
\end{tabular}

$Q$-value of $1 \AA^{-1}$. In Table I values of $\langle\tau\rangle$ and $\beta$ are given for $Q=0.70 \AA^{-1}$. Since $\beta$ is always lowest (i.e., the total relaxation function is most stretched in time) at the lowest $Q$-values where $\langle\tau\rangle$ is largest, the data suggest that $\beta$ is strongly related to the average relaxation time $\langle\tau\rangle$. This is further supported by the finding that $\beta$ also tends to be slightly lower for the samples exhibiting the slowest average dynamics at a given $Q$-value. These observations are expected since the slow longrange diffusion process has a stronger $Q$-dependence and is more sample dependent, than the faster and less $Q$-dependent local relaxations. Thus, the difference in time scale between the long-range diffusion process and the more local motions increases with decreasing $Q$-value and increasing time scale of the long-range diffusion process, and this leads to a more stretched total relaxation function.

The non-monotonic concentration dependence of the dynamics becomes even more evident in Fig. 4, where $\langle\tau\rangle$ is shown as a function of the water concentration for confined 2PGME- $\mathrm{H}_{2} \mathrm{O}$ and $2 \mathrm{PGME}-\mathrm{D}_{2} \mathrm{O}$, as well as the extracted water dynamics. For comparison we also show the corresponding values for the bulk solutions, as presented in Ref. 16. As for the bulk samples it is clear that the water dynamics is faster than the dynamics of 2PGME. However, in the MCM-41 the difference is largest at $75 \mathrm{wt} . \%$ water, rather than at $30 \mathrm{wt} . \%$ as observed for the bulk system, due to the fact that the slowest dynamics of 2PGME has shifted from the solution containing $30 \mathrm{wt} . \%$ water to the solution with $75 \mathrm{wt} . \%$ water in the confinement. This substantial shift will be discussed in Sec. IV.

Examples of typical $Q$-dependences of the dynamics are shown in Fig. 5 for confined solutions of 2PGME with 45 wt. $\% \mathrm{H}_{2} \mathrm{O}$ and $\mathrm{D}_{2} \mathrm{O}$, respectively, as well as for the estimated water dynamics. $\mathrm{I}(Q, t)$ relaxes considerably faster with increasing $Q$, and at the lowest $Q$-values only a small part of the whole relaxation is visible in the experimental time win- dow reaching up to $120 \mathrm{ps}$. This further implies that the error bars of the fit parameters $\tau$ and $\beta$ (and therefore also $\langle\tau\rangle$ ) are largest at the lowest $Q$-values. However, even if these large error bars are taken into account the $Q$-dependence seems to be slightly stronger than $Q^{2}$ in the low Q-range, as shown in Fig. 6 and discussed in more detail for the corresponding bulk solutions in Ref. 16. Since a $Q^{2}$-dependence is a characteristic feature of stochastic translational diffusion it is clear that the

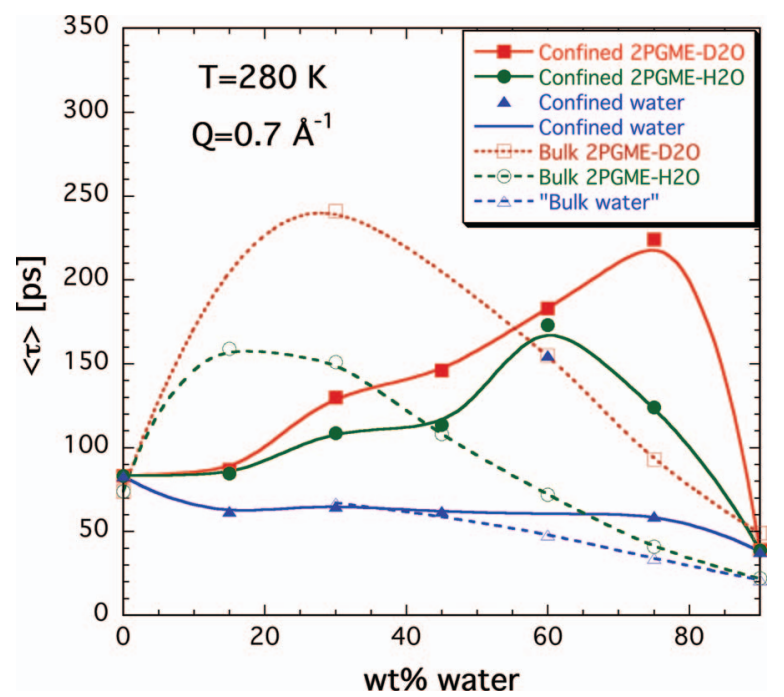

FIG. 4. Average relaxation times, $\langle\tau\rangle$, determined by Eq. (2) from the $\tau$ and $\beta$ parameters obtained from the KWW fits shown in Fig. 3. $\langle\tau\rangle$ as a function of the wt.\% water is shown for confined (solid symbols) and bulk ${ }^{16}$ (open symbols) 2PGME-water solutions of both $\mathrm{H}_{2} \mathrm{O}$ (circles) and $\mathrm{D}_{2} \mathrm{O}$ (squares), as well as the estimated water dynamics at each concentration (triangles), as described in the main text. The lines are guide to the eyes only to make it easier to see the approximative concentration dependences (in this case the estimated water dynamics in MCM-41 at $60 \mathrm{wt}$.\% water has been ignored due to its inconsistency with the data at the other water concentrations). "Bulk water" refers to the concentration dependence of the water dynamics in the bulk solutions of 2PGME-water. 


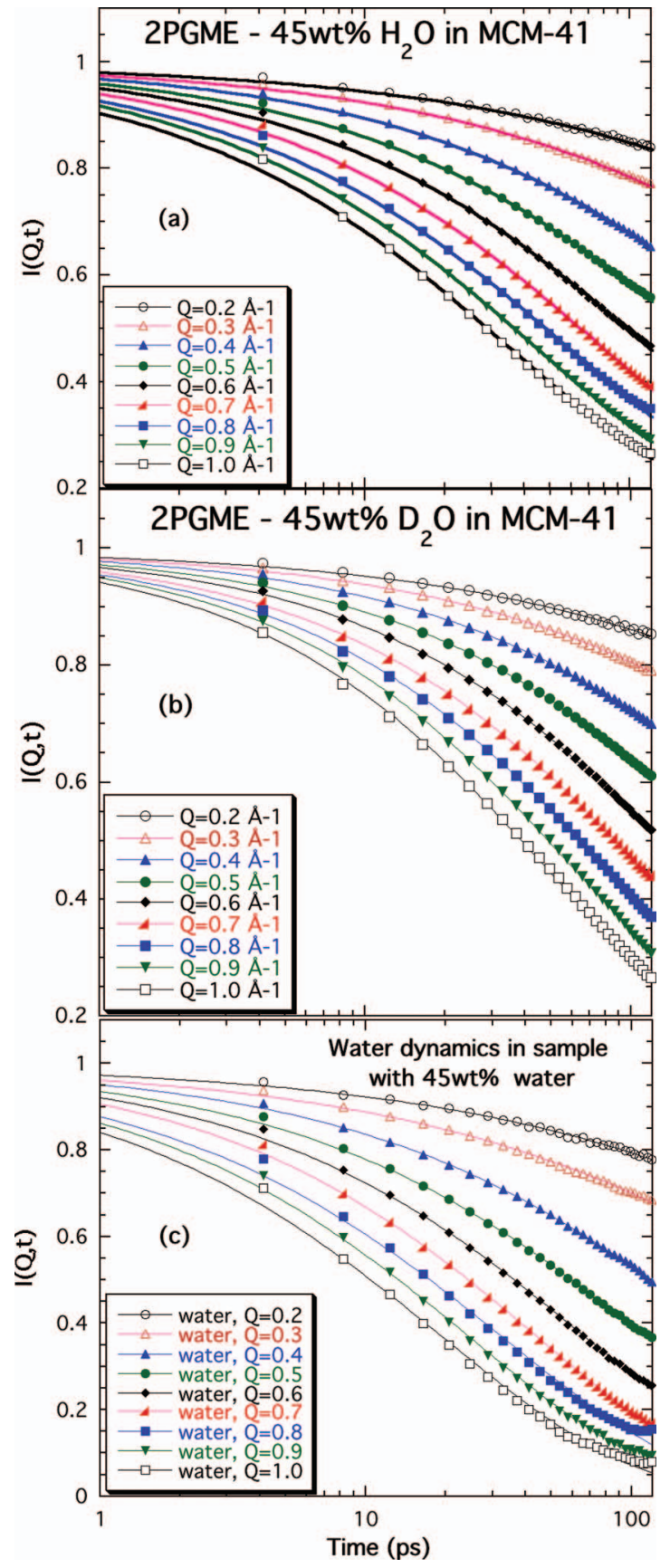

FIG. 5. Intermediate scattering functions, $I(Q, t)$, at $280 \mathrm{~K}$ for (a) confined 2PGME- $\mathrm{H}_{2} \mathrm{O}$, (b) confined 2PGME- $\mathrm{D}_{2} \mathrm{O}$, and (c) the estimated water dynamics in selected composition of 45 wt.\% water. Data are shown for the 9 $Q$-values in the range $0.2-1 \AA^{-1}$, as indicated in the figures, and the solid lines are KWW fits to the data points.

dominating dynamics is of translational diffusion character, rather than of local and/or rotational character. By approximating the overall $Q$-dependences shown in Fig. 6 to $Q^{2}$ the resulting linear fits can be used to estimate the diffusion constants, $D$, by the relation $D=\frac{1}{\langle\tau\rangle Q^{2}} \cdot{ }^{27,28}$ The so obtained diffusion constants are also given in Table I.

\section{DISCUSSION}

Qualitatively, the confined solutions of 2PGME-water behave like the corresponding bulk solutions, with a clear

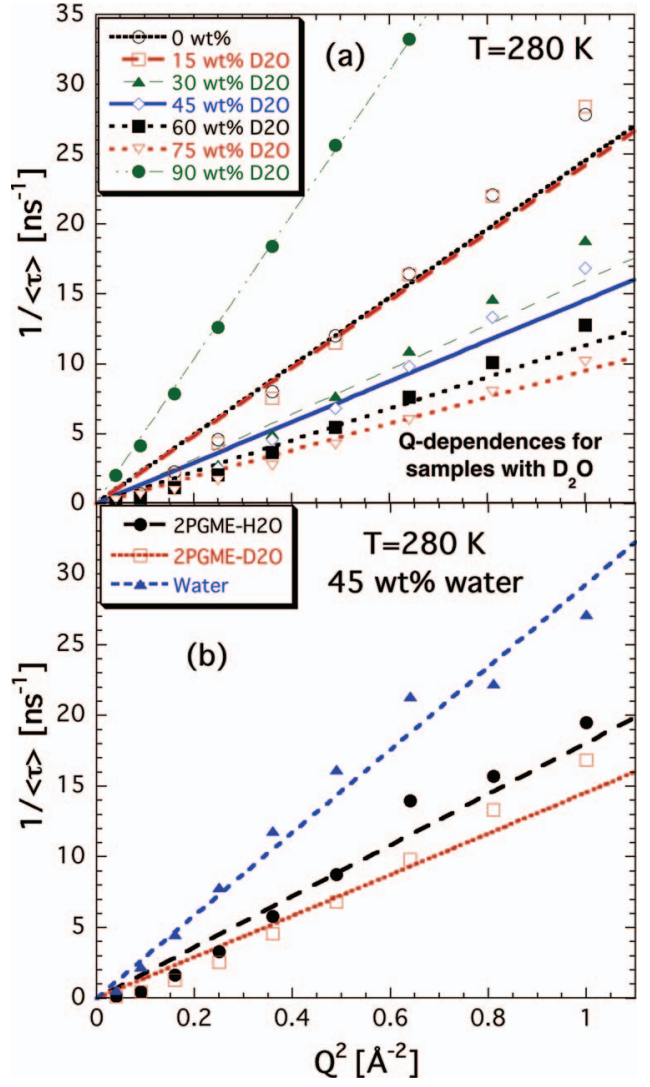

FIG. 6. Reciprocal average relaxation times, $1 /\langle\tau\rangle$, determined by Eq. (2) from the $\tau$ and $\beta$ parameters obtained from the KWW fits shown in Fig. 5, in the case of figure (b) here. In (a) $1 /\langle\tau\rangle$ is shown as a function of $Q^{2}$ for all the confined solutions containing $\mathrm{D}_{2} \mathrm{O}$. The lines indicate the linear fits to deduce the average diffusion constants, $D$, (presented in Table I) of all the confined solutions, as well as the estimated water contribution, at $T=280 \mathrm{~K}$.

non-monotonic concentration dependence of mainly the diffusive dynamics of the 2PGME molecules. However, on a more quantitative level there are substantial differences, where the most prominent difference is that the slowest viscosity related dynamics of $2 \mathrm{PGME}$ is observed at a water concentration of $75 \mathrm{wt} . \%$, to be compared with the $30 \mathrm{wt} . \%$ obtained for the corresponding bulk solutions. Since other studies of aqueous solutions confined in the silica pores of MCM- 41 have indicated ${ }^{18-21}$ that the two liquids becomes at least partly demixed on a nanometer length scale a likely reason for this shift is that some of the water (i.e., the first water added to 2PGME) prefers to hydrate the hydroxyl surface groups of the silica pores, instead of mix with 2PGME. The opposite demixing behavior, with the non-aqueous component hydrogen bonded to the silica surface, ${ }^{20,21}$ seems less consistent with the experimentally observed concentration independent dynamics of 2PGME at low water concentrations. The reason for this concentration independence is more likely that water does not mix with 2PGME at low water concentrations. The concentration dependent dynamics of confined 2PGME$\mathrm{D}_{2} \mathrm{O}$ shown in Fig. 4 indicates that water starts to mix with 2 PGME at a water concentration of about $20 \mathrm{wt} . \%$. Here it should be noted from the $T_{g}$-values presented in Fig. 1 that at such low temperatures the two liquids seem to be more clearly phase separated to much higher water concentrations, 
since a concentration independent $\mathrm{T}_{\mathrm{g}}$ was observed all the way up to $45 \mathrm{wt} \% \%$ water. Furthermore, the slowest dynamics of 2 PGME, caused by the saturation point of bridging water clusters, should also be shifted to a higher water concentration at low temperatures, as indicated by comparing the $\mathrm{T}_{\mathrm{g}}$ values of the bulk solutions shown in Fig. 1 with the relaxation times at $280 \mathrm{~K}$ in Fig. 4, due to the fact that the local motions of the water molecules are slower at low temperatures close to $\mathrm{T}_{\mathrm{g}}$ than at $280 \mathrm{~K}$. An increasing size of the water clusters as well as a more pronounced micro-phase separation of the two liquids at low temperatures are also consistent with diffraction studies of other aqueous solutions of alcohols, such as water-methanol mixtures. ${ }^{29}$ However, above the "critical" water concentration of $20 \mathrm{wt} . \%$ in the confinement at $280 \mathrm{~K}$ there is no indication of that the same type of structural model ${ }^{4}$ as proposed for the corresponding bulk system should not be valid for the confined solutions. Hence, larger hydrogen bonded structural entities of two 2PGME molecules and "bridging" water molecules seem to be formed also in the present cylindrical pores with a diameter of about $28 \AA$.

If we perform a more quantitative comparison of the diffusion constants and average relaxation times between the present confined solutions and the previously measured bulk solutions we see in the case of pure 2PGME that D is lower, $\langle\tau\rangle$ is larger and also $\mathrm{T}_{\mathrm{g}}$ is higher in the confinement. Thus, the diffusion and structural relaxation of 2PGME becomes slower in the silica pores, but due to the immediate growth of larger structural entities when water is added to bulk 2PGME, the dynamics of 2PGME is faster in the confinement already at a few percent added water, as shown in Fig. 4. It is not until a water content of about $60 \mathrm{wt} . \%$ is reached that the dynamics of 2PGME become slower again in the confinement. However, the dynamics of confined 2PGME speeds up rapidly with increasing water content above $75 \mathrm{wt}$. $\%$ water, so at $90 \mathrm{wt} . \%$ water the dynamics of 2PGME in the confinement is at least as fast as in bulk. Regarding the estimated water dynamics it can be seen in Fig. 4 and Table I that it is similar in bulk and confinement up to a water content of about 45 wt.\%. However, it should be noted that in this concentration range the water dynamics in the confined and bulk solutions is about 8 times slower than bulk water. ${ }^{30-32}$ This implies that the average dynamics of water at the pore surfaces is about equally slowed down as the water bridging between two 2PGME molecules. A difference in the water dynamics between the confined and bulk solutions can only be observed at higher water concentrations, where the additional water in the confinement participates in the larger structural entities, whereas in the bulk solutions this additional water will be rather "free" and more bulk-like, and therefore move faster. Thus, in the concentration range up to $20 \mathrm{wt} \%$ water, the water is slowed down by surface interactions, and in the concentration range up to 75 wt.\% water the additional water is almost as slow due to its participation in the larger structural entities. It is not until even higher water concentrations are reached additional water is "free" and moves almost as in bulk water. This implies that the water in the silica pores can be divided into three categories; surface water, water bridging between two 2PGME molecules, and rather "free" and bulk-like water. However, since the dynamics of water molecules bridging between two
2PGME molecules is considerably faster than the dynamics of the 2PGME molecules, as shown in Fig. 4 for both confined and bulk solutions, it is clear that these water molecules are moving internally, i.e., changing places, in the formed structural entities on a faster time scale than the whole entities are moving. This faster "internal" water dynamics is caused by the short lifetime of each hydrogen bond, which facilitates diffusion of water within the hydrogen bonded clusters. This "internal" water dynamics is therefore the main reason for why the dynamics of $2 \mathrm{PGME}$ and water have very different concentration dependencies.

In Fig. 4 it can also be seen that the difference between the average relaxation times of the two confined solutions containing $60 \mathrm{wt} . \%$ water is unexpectedly small. Due to the used subtraction procedure to deduce the water dynamics this small difference also makes the average relaxation time of the estimated water dynamics twice as long compared to all the other water concentrations (see Table I). The results suggest that the two samples cannot be structurally equal and that they have slightly different water distributions

From the $Q$-dependences of the dynamics shown in Fig. 6 it is clear that the nature of the molecular motions is preserved in the confinement, since the corresponding bulk solutions show almost identical $Q$-dependences. ${ }^{16}$ Thus, from the experimental data there is no indication for that the confinement to cylindrical pores of a diameter of $28 \AA$ has made the dynamics more localized on the approximate experimental length scale of less than $20 \AA$. The overall dynamics of both 2 PGME and water is clearly dominated by translational diffusion.

\section{CONCLUSIONS}

The present QENS study of 2PGME-water solutions confined in $28 \AA$ pores of MCM-41 has shown several anomalous effects. First, the dynamics of 2PGME exhibits a clearly nonmonotonic dependence on the water concentration, indicating a behavior far from an ideal solution. Second, the slowest dynamics of $2 \mathrm{PGME}$ is shifted to a higher water concentration, in comparison to the corresponding bulk solutions, which suggests that all water is not fully mixed with 2PGME in the silica pores. Most likely, water has a stronger preference to coordinate to the hydrophilic hydroxyl surface groups of the silica pores, leaving the 2PGME molecules in the more central part of the pores. Only at higher water contents there is a sufficient amount of water to partly mix with 2PGME. This mixing gives rise to a non-monotonic concentration dependence of the dynamics of 2PGME, due to the same formation of large structural entities of hydrogen bonded water and 2PGME molecules as observed for the corresponding bulk system. ${ }^{16}$ Thus, the present geometrical confinement does not seem to affect the ability to form such hydrogen bonded entities of water and 2PGME molecules, but the MCM-41 nevertheless affects the hydrogen bonding in the solutions due to the hydrogen bonding of water to the pore surface, and this causes the alteration of the concentration dependent dynamics of the confined solutions. 


\section{ACKNOWLEDGMENTS}

This work was financially supported by the Swedish Research Council and the Swedish Energy Agency.

${ }^{1}$ J. Mattsson, R. Bergman, P. Jacobsson, and L. Börjesson, Phys. Rev. B 79, 174205 (2009)

${ }^{2}$ A. Bernson and J. Lindgren, Polymer 35, 4842 (1994).

${ }^{3}$ J. Sjöström, J. Mattsson, R. Bergman, E. Johansson, K. Josefsson, D. Svantesson, and J. Swenson, Phys. Chem. Chem. Phys. 12, 10452 (2010).

${ }^{4}$ J. Sjöström, J. Mattsson, R. Bergman, and J. Swenson, J. Phys. Chem. 115, 10013 (2011).

${ }^{5}$ M. Ageno and C. Frontali, Proc. Natl. Acad. Sci. U.S.A. 57, 856 (1967).

${ }^{6}$ S. Akhtar, M. M. H. Bhuiyan, M. S. Uddin, B. Sultana, M. Nessa, and M. A. Saleh, Phys. Chem. Liq. 37, 215 (1999).

${ }^{7}$ K. A. Valiev and M. I. Emel'yanov, Zh. Strukt. Khim. 5, 7 (1964)

${ }^{8}$ A. K. Soper and J. L. Finney, Phys. Rev. Lett. 71, 4346 (1993).

${ }^{9}$ D. T. Bowron, A. K. Soper, and J. L. Finney, J. Chem. Phys. 114, 6203 (2001)

${ }^{10}$ S. Dixit, J. Crain, W. C. K. Poon, J. L. Finney, and A. K. Soper, Nature (London) 416, 829 (2002)

${ }^{11}$ M. Misawa, Y. Inamura, D. Hosaka, and O. Yamamuro, J. Chem. Phys. 125, 074502 (2006).

${ }^{12}$ M. Misawa, K. Yoshida, K. Maruyama, H. Munemura, and Y. Hosokawa, J. Phys. Chem. Solids 60, 1301 (1999).

${ }^{13}$ V. Calandrini, A. Deriu, G. Onori, R. E. Lechner, and J. Pieper, J. Chem. Phys. 120, 4759 (2004).

${ }^{14}$ M. Nakada, K. Maruyama, O. Yamamuro, and M. Misawa, J. Chem. Phys. 130, 074503 (2009).

${ }^{15}$ G. C. Benson and O. Kiyohara, J. Solution Chem. 9, 791 (1980).
${ }^{16}$ J. Swenson, J. Sjöström, and F. Fernandez-Alonso, J. Chem. Phys. 133, 234506 (2010).

${ }^{17}$ Biophysics of Water, edited by F. Franks and S. Mathias (Wiley, London, 1983).

${ }^{18}$ S. Kittaka, M. Kuranishi, S. Ishimaru, and O. Umahara, J. Chem. Phys. 126, 091103 (2007).

${ }^{19}$ K. Elamin, H. Jansson, S. Kittaka, and J. Swenson, Phys. Chem. Chem. Phys. 15, 18437 (2013).

${ }^{20}$ A. Lerbret, G. Lelong, P. E. Mason, M.-L. Saboungi, and J. W. Brady, J. Phys. Chem. B 115, 910 (2011).

${ }^{21}$ X.-Y. Guo, T. Watermann, and D. Sebastiani, J. Phys. Chem. B 118, 10207 (2014).

${ }^{22}$ J. Sjöström, R. Bergman, C. Wadell, T. Moberg, J. Swenson, and J. Mattsson, J. Phys. Chem. B 115, 1842 (2011).

${ }^{23}$ T. Unruh, J. Neuhaus, and W. Petry, Nucl. Instrum. Methods Phys. Res., Sect. A 580, 1414 (2007).

${ }^{24}$ C. J. Carlile and M. A. Adams, Physica B 182, 431 (1992).

${ }^{25}$ G. Williams and D. Watts, Trans. Faraday Soc. 66, 80 (1970).

${ }^{26}$ G. W. Sherer, J. Non-cryst. Solids 123, 75 (1990).

${ }^{27}$ M. Bée, Quasielastic Neutron Scattering: Principles and Applications in Solid State Chemistry, Biology and Material Science (Adam Hilger, Bristol, Philadelphia, 1988).

${ }^{28}$ S. Longeville, W. Doster, M. Diehl, R. Gähler, and W. Petry, in Neutron Spin Echo Spectroscopy, edited by F. Mezei, C. Cappas, and T. Gutberlet (Springer-Verlag, Berlin, 2003), p. 325.

${ }^{29}$ L. Dougan, R. Hargreaves, S. P. Bates, J. L. Finney, V. Réat, A. K. Soper, and J. Crain, J. Chem. Phys. 122, 174514 (2005).

${ }^{30}$ R. J. Hunter, G. C. Stirling, and J. W. White, Nature (London) 230, 192 (1971).

${ }^{31}$ J. J. Tuck, P. L. Hall, M. H. B. Hayes, D. K. Ross, and C. Poinsignon, J. Chem. Soc., Faraday Trans. 80, 309 (1984).

${ }^{32}$ J. Teixeira, M.-C. Bellissent-Funel, S. H. Chen, and A. J. Dianoux, Phys. Rev. A 31, 1913 (1985). 